Conclusion The presented study describes applicability for PACT in the successful treatment of highly resistant CD spores using a two-phase antimicrobial approach and that taurocholic acid is nontoxic to humans. This strategy could be effective at reducing the significant numbers of patients with relapsing $\mathrm{CD}$, the length of stay for these patients, associated morbidity as well as the potential mortality of $\mathrm{CD}$ which mostly arises from this sub-group of patients.

Disclosure of Interest None Declared.

\section{PTH-008 PHOTODYNAMIC ANTIMICROBIAL CHEMOTHERAPY (PACT) SELECTIVELY KILLS CLOSTRIDIUM DIFFICILE OVER COLON CELLS AND IS EFFECTIVE AGAINST 5 HYPERVIRULENT STRAINS OF THE PATHOGEN}

doi:10.1136/gutjnl-2013-304907.496

\begin{abstract}
1,2, $\mathrm{M}$ A Butt, ${ }^{1,3 \mathrm{~L}}$ De Sordi, ${ }^{4} \mathrm{G}$ Yahioglu, ${ }^{1} \mathrm{C}$ Moose, ${ }^{5,6} \mathrm{~S}$ Battah, ${ }^{4}$ I Stamati, ${ }^{4} \mathrm{M}$ Deonarain, ${ }^{3} \mathrm{P}$ Mullany, ${ }^{3} \mathrm{E}$ Allan, 1,2L B Lovat. 'National Medical Laser Centre; ${ }^{2}$ Gastroenterology;

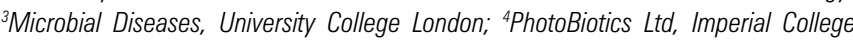
London, London; ${ }^{5}$ Organix Ltd; ${ }^{6}$ Department of Biological Sciences, University of Essex, Colchester, UK
\end{abstract}

Introduction Clostridium difficile (CD) is the leading cause of hospital and community-acquired antibiotic-associated diarrhoea in the developed world. Since 2003, a new lineage of strains with more severe virulence has emerged, leading to an increased number of outbreaks of disease in North America and Europe and raising the impellent need for an effective therapy. Photodynamic Antimicrobial Chemotherapy (PACT) utilises the ability of light-activated photosensitisers (PS) to produce free radical species lethal to the target pathogens. To date, no pathogens have developed resistance to PACT. This study aimed to develop and evaluate PACT for the treatment of $C D$. Methods High throughput screening of 15 photosensitiser (PS) drugs were performed in aerobic conditions against the hypervirulent R20291 strain of $C D$. These included both clinically approved PS drugs and experimental PS's engineered for CD. Lead candidate agents were then tested against C. difficile strain R20291 in microaerophilic and anaerobic conditions, against 4 of the other most clinically significant hypervirulent $C D$ strains, each belonging to a different ribotype, and against the human colonic cell line HT-29 at effective antimicrobial doses to exclude background colonic cytotoxicity.

Results Nine PS were successful in killing $99.99 \%$ of R20291 at a concentration of $10 \mu \mathrm{M}$ after exposure to laser light at $665 \mathrm{~nm}$ at an intensity of $24 \mathrm{~mJ} / \mathrm{cm} 2$. Remarkably, three of them (S4, CE6 and PS4) also reduced bacterial growth by $99.9 \%$ in absence of oxygen at the concentration of $50 \mu \mathrm{M}$ and no PS-associated toxicity was observed in the absence of light. PACT was found to be similarly effective against all 5 hypervirulent $C D$ strains. Three PS were not toxic to HT-29 cells at effective antimicrobial concentrations.

Conclusion We have found PACT effectively kills the 5 most clinically relevant hypervirulent $C D$ strains. PACT efficacy traditionally is thought to require oxygen to generate reactive oxygen species. We have shown PACT to be effective in anaerobic conditions mimicking the colonic microenvironment in which $C D$ reside. As PACT was not toxic to human HT-29 cells at effective antimicrobial doses, this would permit selective targeting of the pathogen in the site of infection. It is believed the research being undertaken could be an important step towards the eradication of $C$. difficile colitis.

Disclosure of Interest None Declared.

\section{PTH-009 ENDOSCOPISTS WHO ARE METICULOUS IN THEIR CAECAL IMAGE DOCUMENTATION DETECT MORE POLYPS}

doi:10.1136/gutjnl-2013-304907.497

1." M H Thoufeeq, 'B Rembacken. 'Endoscopy/Gastroenterology, LEEDS TEACHING HOSPITALS, Leeds, UK
Introduction Adenoma detection is now accepted as an important quality indicator of colonoscopy. There is a significant variation in polyp detection rates among colonoscopists. Although the ESGE has recommendations regarding quality of images taken during endoscopy, there are no studies looking at the quality of caecal images versus outcomes of the procedures.

Here we tested our hypothesis of endoscopists who have better quality image documentation of the caecum have higher polyp detection.

Methods This retropective study was between June 2011 and May 2012. Planned colonoscopies performed by 16 experienced colonoscopists were included.

We excluded procedures with planned therapeutic procedures, inadequate bowel preparation, rectal hyperplastic polyps, bowel cancer screen colonoscopies, previous hemicolectomy and incomplete procedures.

The pre-procedure data collected were age and gender of patients indication of procedures. The intra-procedure data collected were duration of the procedure, number of images stored in the endoscopy database, quality of caecal image, number of polyps (excluding rectal hyperplastic polyps). We cross-checked our pathology database to confirm histology of the polyps.

We formulated a new scoring system, caecal image documentation score (CIDS). The CIDS was as follows; no image $=0$, unclear image $=1$, clear image $=2$ and clear image with a label $=3$.

Results A total of 651 procedures performed by 16 colonoscopists were analysed. The mean number of procedures performed by each colonoscopist was 41. Mean age of the patients was 60.3 years. $46 \%$ of the patients were males. The mean CIDS for the 16 endoscopists was 2.13 . The mean polyp detection rate (PDR) was $24 \%$ and mean polyp per procedure (PPP) was 0.42 .

Colonoscopists with mean CIDS $>2.0$ ( $n=429$ procedures, 10 colonoscopists) had PDR of $28 \%$ and PPP of 0.52 . On the other hand, 6 colonoscopists (222 procedures) with mean CIDS of $<2.0$ had PDR of $16 \%$ and PPP of 0.24 .

Mean CIDS $>2.0$ was associated with greater PDR (OR 2.1, CI $1.4-3.2 p=0.001$ ). When adjusting for age, gender, and indication for colonoscopy, the mean CIDS $>2.0$ remained an independent predictor of greater PDR, OR 2.4, 95\% CI $1.5-3.8 p<0.001$

Mean CIDS $>2.0$ was associated with greater right-sided polyp detection rate, OR 3.4, CI $1.9-6.6 \mathrm{p}<0.001$. When adjusting for age, gender, and indication for colonoscopy, the mean CIDS $>2.0$ remained an independent predictor of greater right-sided PDR, OR $4.0,95 \%$ CI $2.2-8.1 \mathrm{p}<0.001$.

\section{Abstract PTH-009 Table 1}

\begin{tabular}{lcc}
\hline & $\begin{array}{c}\text { Polyp per } \\
\text { procedure }\end{array}$ & $\begin{array}{c}\text { Polyp detection } \\
\text { rate (PDR) }\end{array}$ \\
\hline Colonoscopists with mean CIDS $>2.0$ & 0.52 & $28 \%$ \\
Colonoscopists with mean CIDS $<2.0$ & 0.24 & $16 \%$ \\
\hline
\end{tabular}

Conclusion Colonoscopists who are more meticulous in caecal image documentation detect more polyps per procedure and have higher polyp detection rates. Better caecal image documentation also improves right colonic polyp detection.

Disclosure of Interest None Declared.

\section{PTH-010 RISES IN BOTH WHITE CELL COUNT AND CRP AT DAY 3 PREDICT FAILURE OF TREATMENT WITH METRONIDAZOLE IN C.DIFFICILE INFECTION}

doi:10.1136/gutjnl-2013-304907.498

1.*N M Joshi, 'K Kok, 'W Alazawi, 'J Goodhand, ${ }^{2} \mathrm{~S}$ S Das, 'D S Rampton. 'Centre for Digestive Diseases, Barts and The London School of Medicine and Dentistry, ${ }^{2}$ Department of Medical Microbiology, Barts Health NHS Trust, London, UK 